\title{
Position Location Technique in Wireless Sensor Network Using Rapid Prototyping Algorithm
}

\author{
Touati Youcef, Aoudia Hania, Ali-Cherif Arab and Mohamed Demri \\ University of Paris 8 at Vincennes/Saint-Denis \\ Computer Science and Artificial Intelligence Lab. LIASD
}

France

\section{Introduction}

Localization process using Wireless Sensor Network (WSN) is one of the main services that have been prospected in order to ensure assisted people a better mobility and assistance in their life. In this direction, localization methods have been investigated (Röhring, 2009; Vossiek, 2003) and can be classified as the used information, such as: Connectivity information, Angle of Arrival (AOA), Time of Arrival (TOA), Time Difference of Arrival (TDOA), and RSSI method. The latter is the most used one, and it consists to acquire a distance from measuring the received signal strength of the incoming radio signal. The idea behind RSSI is that the configured transmission power at the transmitting device directly affects the receiving power at the receiving device. In practical scenarios, the ideal distribution of the received power is not applicable, because the propagation of the radio signal is interfered with a lot of influencing effects, such as: reflection on metallic objects, superposition of electromagnetic fields, noises and so on. Implementing these models requires parameters identification phase, allowing being closer to sensor model. In reality, it represents just an approximation procedure which doesn't reflect a real world.

Several sensor models have been developed (Tsung-Han, 2008; Awad, 2007; Graefenstein, 2008; Sugano, 2006; Cho, 2007). The most used one can be described by the following equation:

$$
P(d)=P\left(d_{0}\right)-10 \alpha \log \frac{d}{d_{0}}
$$

Where $d_{0}, P\left(d_{0}\right)$ and $\alpha$ are respectively the distance of reference, the received power and an approximation factor $(\approx 3.5)$.

Another methods based on Link Quality Indicator property (LQI) have been proposed too for distance calculation. That property is considered as a measure of force where the quality of received parquets is proportional to RSSI signal. Thus, the distance between nodes can be determined as follows:

$$
P_{i}(x, y)=\frac{\sum_{j=1}^{n}\left(w_{i j} \cdot B_{j}(x, y)\right)}{\sum_{j=1}^{n} w_{i j}}
$$


Where $B(x, y)$ is the position of anchors and $w_{i j}$ is the link weight between nodes, such as:

$$
w_{i j}=\frac{1}{\left(d_{i j}\right)^{\beta}}
$$

Parameter $\beta$ is experimentally estimated.

These methods are very attractive nevertheless they present some drawbacks on requiring theoretical or generic sensory models closer to the real ones. Moreover, these models require a mass of information to achieve higher accuracy leading to increase traffic, sensors energy consumption and a better approximation and optimization of parameters taking into account environment change and signal failures. Thus, it seems to be difficult and less efficient to implement. A good localization algorithm should calculate a position as fast as possible and should be resistant to environmental influences as well as imprecise distances. The challenge here is to prospect others approaches offering more realistic models taking into account these constraints and particularly parameters approximation problem.

Introduced by Zadeh (Zadeh, 1965) in 1965, fuzzy logic concept has been widely applied in different applications such as control and identification process, pattern recognition, graphics analysis and synthesis and robotics (Bezdek, 1981; Dave, 1990; Liang, 2003; Huang, 2003; Lei, 2001) and satisfactory results have been obtained. In contrast, few researches have been directed towards development of fuzzy logic-based approaches for localization in WSN, even if they present advantages in terms of robustness in spite of noise or other sensor failures, ability to handle non-linearity without degradation and the use of linguistic adjectives and relations similar to a natural language for interpretability.

For this purpose, we propose a WSN localization method based on IEEE 802.15.4 standard using fuzzy logic concepts with an evolutionary algorithm for parameters approximation and optimization. The approach allows approximating a realistic model for distance estimation from a set of RSSI measures and taking into account environment influences and signal failures as well as imprecise distances. The approach is based on a FIS optimization methodology combining a Rapid Prototyping (RP) mechanism and Solis and wets' monoagent evolution strategy (Solis, 1981; Glorennec, 1999; Hart, 1994). The methodology allows, in term of probability, a randomized hill-climber with an adaptive step size and a global minima convergence of a coast function. It describes a class of local and global search algorithms with proofs of convergence in the limit of infinite search time.

In contrast to the existing methods for FIS optimization methodology () where a large number of parameters has to be tuned leading to significant computing times requiring high memory capacity (Imai et al., 1998; Jiang et al., 2008; Ramon et al., 2008; Castillo et al., 2008), the proposed approach needs only a few rules for FIS knowledge base optimization, which leads to increased optimization convergence speed and avoids additional criteria such as rules simplification.

The proposed approach takes into account an online and offline aspects and operates in two stages. In the first one, an evolutionary method based on Solis and Wets algorithm is implemented in conjunction with a PA mechanism to estimate online distances between nodes according to a set of RSSI measures. It's based on Glorennec's methodology for FIS data structure optimization and can be implemented off- or on-line and it proceeds as follows: A first set of rules is extracted automatically from training data using RP mechanism Thus, in conjunction with RP mechanism, an evolutionary algorithm based on Solis and Wets method for MFs optimization is then implemented. In the second stage, to 
increase the optimization quality, rules conclusions are updated online using a stochastic gradient method. In order to compute real positions, a triangulation method based on $\mathrm{Al}$ Kash $i$ theory is implemented using data derived from the second phase.

This paper is organized as follows: In Section 2, we present the proposed WSN localization approach based fuzzy logic concepts. Thus, in this context, an evolutionary method based on Solis and Wets algorithm is implemented in conjunction with a PA mechanism to estimate online distances between nodes according to a set of RSSI measures. It's based on Glorennec's methodology for FIS data structure optimization and can be implemented offor on-line. In order to compute real positions, a triangulation method based on Al-Kashi theory is implemented. Section 3 describes the system overview which consists in a smart wheelchair developed in our Lab., called LIASD-WheelChair. In Section 4, experimental and simulation results are presented and discussed. Finally, conclusion and some perspectives will be dressed in section 5 .

\section{Fuzzy localization approach in wireless sensor network}

Implementing our approach requires a FIS architecture which consists on a set of Multiinputs/Single-output (MISO) FIS modules (Cho, 2007). The number of the FIS modules can be determined according to output vector dimension. Each FIS module is a zero-order Takagi-Sugeno Fuzzy Model, also known as TSFM (Takagi \& Sugeno, 1987). The architecture is illustrated in Fig.1.

Each FIS module takes the antecedents $I_{\text {RSSI }}$ at its inputs and produces a distance estimation $d_{f_{-} R S S I_{k}}$, where $k=1, \cdots, n$, with $n$, number of FIS modules.

As in TSFM, the architecture implements rules of the following form:

IF IRSSI is $A(j)\left(I_{R S S I 1}\right)$ and ... I IRSSii is $A(j)\left(I_{R S S I i}\right)$ THEN $d_{f_{-} R S S I i}=f(j)\left(I_{R S S I}\right)$

$I_{R S S I}$ is the normalized input and $A(j)\left(I_{R S S I}\right)$ is a $k$-dimensional fuzzy set of the $j^{\text {th }}$ rule $(j=1, \cdots, m)$.

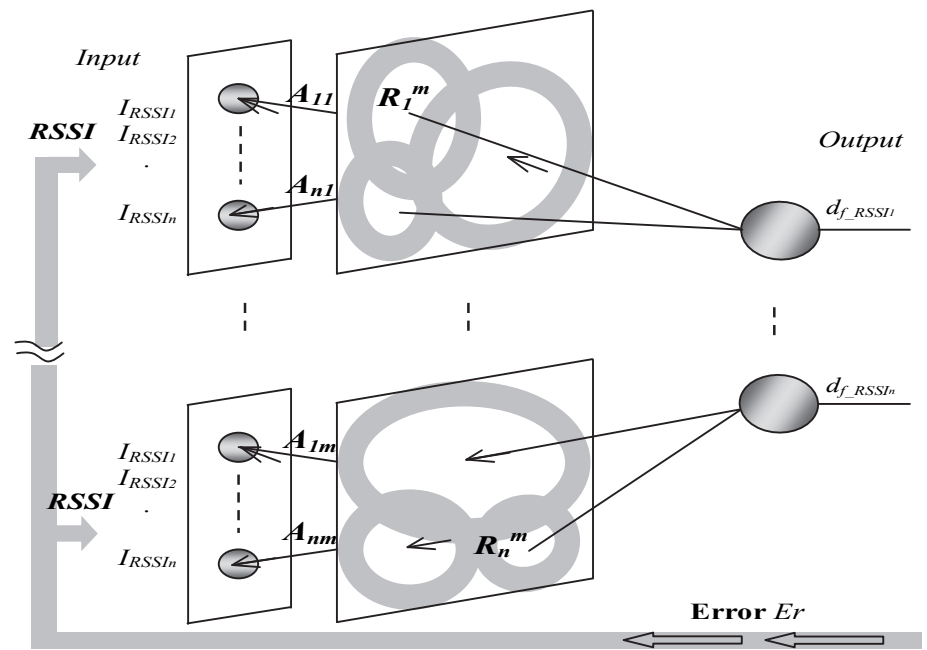

Fig. 1. Takagi-Sugeno fuzzy model 
The output $d_{f_{-} R S S I i}$ which is defined for each rule is computed as a weighed average of the contribution of each rule, so that:

$$
d_{f_{-} R S S I}=\frac{\sum_{j=1}^{L} w^{(j)}\left(I_{R S S I}\right) \cdot y^{(j)}}{\sum_{j=1}^{L} w^{(j)}\left(I_{R S S I}\right)}
$$

$L$ is the number of rules, $w^{(j)}$ and $y^{(j)}$ are the degree of truth and the conclusion of the $j^{\text {th }}$ rule respectively.

For the $i^{\text {th }}$ input variable $I_{R S S I}$, the domain of validity is divided into $k_{i}$ fuzzy sets labelled $A_{i}^{k_{i}}\left(I_{R S S I_{i}}\right)$, as shown in Fig.2. A small value of $k_{i}$ is sufficient, leading to an increased convergence speed of optimization procedure. Here, each fuzzy subset is characterized by a modal value $m_{i, k i}$ with $k_{1}=5$.

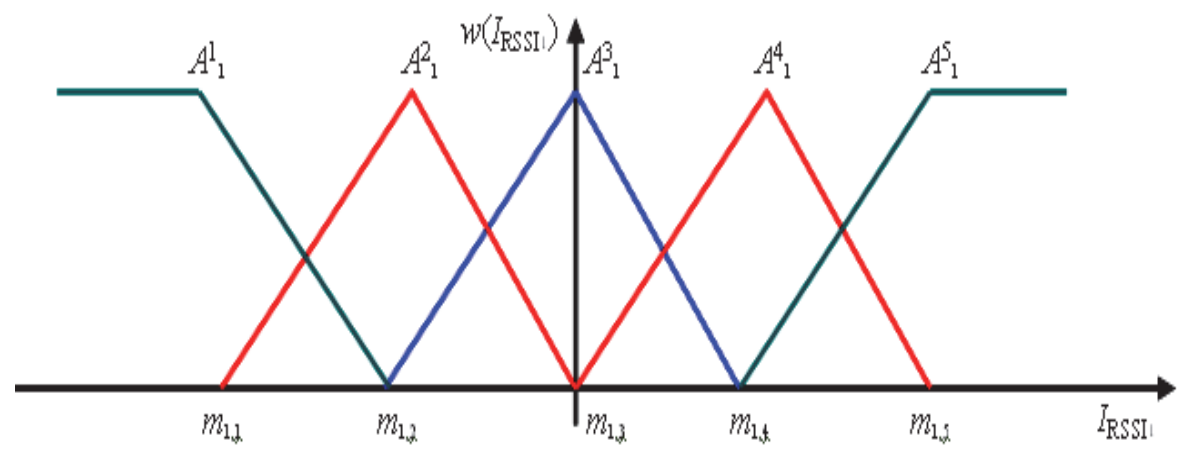

Fig. 2. Fuzzy partitioning

The degree of truth for each input $I_{R S S I i}$ can be written as follows:

$$
w^{(j)}\left(I_{R_{S S I}}\right)=\max \left(1-\frac{\left|I_{R S S I_{i}}-a_{k_{i}}^{(j)}\right|}{b_{k_{i}}}, 0\right)
$$

So that $a_{k_{i}}^{(j)}=\frac{j-1}{k_{i}-1}$ and $b_{k_{i}}=\frac{1}{k_{i}-1},\left(j=1, \ldots, k_{i}\right)$

Usually $f(j)\left(I_{R S S I}\right)$ is a polynomial function in the input variables $I_{R S S I i}$, but it can be any function as long as it can appropriately describe the output of the system within the fuzzy region specified by the antecedent of the rule. When $f($.$) is a first-order polynomial, the$ resulting FIS is called a first-order Sugeno fuzzy model, which was originally proposed in (Takagi \& Sugeno, 1987). When $f($.$) is a constant, we have a zero-order Sugeno FIS, which is$ similar to a Mamdani FIS case where each rule's consequent is specified by a fuzzy singleton. 


\subsection{Fuzzy estimator for RSSI based-localization}

In this section, an evolutionary method based on Solis and Wets algorithm is implemented in conjunction with a PA mechanism (Solis, 1981; Glorennec, 1999) to estimate online distances between nodes according to a set of RSSI measures. It's based on Glorennec's methodology for FIS data structure optimization (Glorennec, 1999) and can be implemented off- or on-line and it proceeds as follows: A first set of rules is extracted automatically from training data using Rapid Prototyping (RP) mechanism Thus, in conjunction with RP mechanism, an evolutionary algorithm based on Solis and Wets method for MFs optimization is then implemented.

\subsubsection{RP mechanism for initial knowledge base extraction}

The FIS architecture is based on the Takagi-Sugeno (TS) model (Lei, 2001; Takagi, 1987) and consists of several FIS' modules. It's used to learn first the FIS from the measured data set, as it's illustrated in Fig.3.

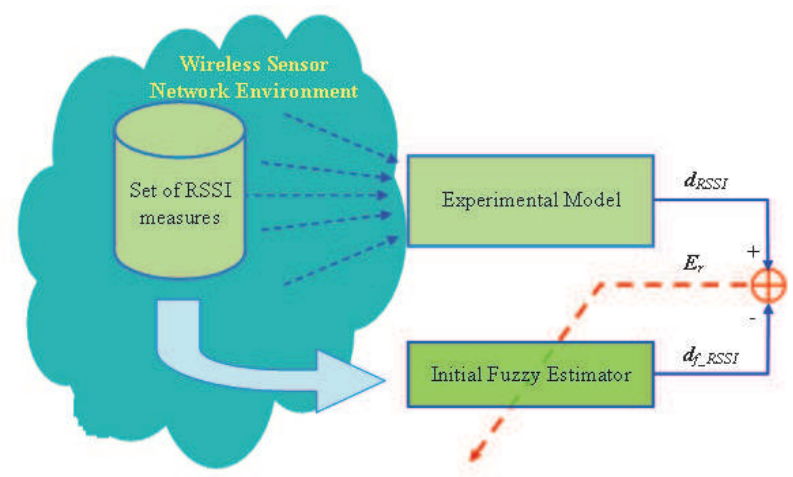

Fig. 3. Initial fuzzy database construction

The first objective is to build a FIS so that for an input $I_{R S S I}$, it produces an output $d_{f_{-} R S S I_{i}}$ that approximates the real measured values $d_{R S S I_{i}}$, obtained from MICAz sensors, minimizing the total Mean Squared Error (MSE) $E_{r}$ as follows:

$$
E_{r}=\frac{1}{2} \sum_{\mu}\left(d_{R S S I i}^{\mu}-d_{f_{-} R S S I i}{ }^{\mu}\right)^{2}
$$

$\mu$ is the index labelling the training samples.

Thus, from an initial learning database which is generated from a set of RSSI measures is then used by a RP mechanism in order to produce initial rules. This algorithm uses a MSE given in (6).

When the learning point is close to a node, the corresponding rule conclusion corresponds to a better approximation of the desired output. Using training data, FIS is initialized availing the property of a strong fuzzy partition, i.e., rule conclusion is the image of one of its nodal vectors.

Thus, if $m(j)$ is a nodal vector corresponding to the $j^{\text {th }}$ rule and $y^{(j)}$ is a conclusion, we have: $F I S(m(j)))=y^{(j)}$ where $F I S(\cdot)$ is the input/output function of the FIS. 
The objective is to find a solution which is already close to the optimal one and to accelerate the training phase. To initialize a vector $y(j)$, we use the continuity of FIS, defined as follows:

$$
\forall j, \lim _{x \rightarrow m^{(j)}} \operatorname{FIS}(x)=y^{(j)}
$$

For a couple of elements $(x, y)$ of training set $S$, the closer $x$ is to $m^{(j)}$, the closer $y$ must be to $y^{(j)}$. This latter, can be approximated by $y^{*}$, so that the vector of the training base $\left(x^{*}, y^{*}\right)$ can be is defined as follows:

$$
x^{*}=\arg \min _{x \in S}\left\|x-m^{(j)}\right\| \equiv \arg \max _{x \in S} w^{(j)}(x)
$$

To limit the noise effects of the learning data, the contribution of the closest points of each node is weighted as follows:

$$
y^{(j)}=\sum_{w^{(j)} \geq s}\left(w^{(j)}(x)\right)^{m} \cdot y(x)
$$

$s$ is a given threshold and $m$ is a positive integer.

The algorithm for initializing rules conclusions and input MFs can be summarized as follows:

For each rule $j$ do:

Search $\left(x^{*}, u_{x}^{*}\right)$ in A so that:

$$
\begin{aligned}
& w_{j}\left(x^{*}\right)=\max _{x \in A} w_{j}(x) \\
& y^{j}=u_{x}^{*}
\end{aligned}
$$

End

To limit the noise effects of the learning data, the contribution of the points closest to each node is weighed by RPA, as follows:

$$
y^{i}=\sum_{w_{i}(x) \geq s} w_{i}^{m}(x) \times u_{x}
$$

Here, $s$ is a data threshold and $m$ an integer higher than 1 .

The obtained conclusion remains then in the convex envelope of possible values, which is not guaranteed by gradient descent algorithms.

Thus, once initial knowledge base is extracted, in conjunction with RP mechanism, we implement an evolutionary algorithm based on Solis and Wets method for MFs optimization.

\subsubsection{Evolutionary algorithm based on Solis and Wets method for MFs optimization}

The MFs optimization reduces to the optimization of the nodal values. The input variable for the optimization algorithm is the set of the nodal values for each input. Thus, each FIS module can be coded as:

$$
A=\left(m_{1,1}, \cdots, m_{1, k_{1}}, m_{2,1}, \cdots, m_{2, k_{2}}, \cdots, \cdots, m_{n, 1}, \cdots, m_{n, k_{n}}\right)
$$


Where $m_{i, l}$ represents the $l^{\text {th }}$ nodal value at $i^{\text {th }}$ dimension, with the constraint that for every $i$ : $m_{i, 1}<m_{i, 2}<\cdots<m_{i, k_{i}}$.

Each step of the proposed algorithm starts with some initial point $A^{(0)}$ with mean $M^{(0)}$ and $t=0$. Note $E(x)$ the objective function.

Each step starts with a current point or initial vector $A^{(0)}$ created by dividing each input variable domain into equal parts.

A deviate $G^{(k)}$ is chosen from a normal distribution whose standard deviation is given by parameter $\rho$.

If either $A^{(k)}-G^{(k)}$ or $A^{(k)}+G^{(k)}$ is better, a move is made to the better point and a "success" is recorded. Otherwise a "failure" is recorded. After several successes in a row, $\rho$ is increased to move more quickly. After several failures in a row, $\rho$ is decreased to focus the search. Additionally, a bias term is included to give the search momentum in directions that yield success.

Correspondent FIS is evaluated along learning set using for example, criteria $E_{r}=\frac{1}{2} \sum_{\mu}\left(d_{\text {RSSI }} \stackrel{\mu}{ }-d_{f_{-} R S S I} i^{\mu}\right)^{2}$, which depends on $A^{(0)}$, then we have : $E\left(A^{(0)}\right)=E_{r}$.

A Gaussian noise is then added to $A^{(0)}$ components, which lead to modify MF. If the vector doesn't verify the imposed constraints, a new pulling is computed until obtaining of a conformed vector and the evaluation can be restarted.

The objective of vector $M$ is to memorize the good exploration directions in the space. It introduces a bias in the exploration. In the case of failure, this bias is exponentially forgotten: The algorithm tries to follow a valleys to rich the minimum. The Gaussian noise, which is very expansive in term of computation time, is re-used in the direction $A^{(k)}-G^{(k)}$ if the direction $A^{(k)}+G^{(k)}$ is failed. An important feature of this type of local search is that it doesn't rely on gradient information.

The pseudo-algorithm of Solis and Wets local search implementation is given on Fig.4.

\subsection{Fuzzy rules consequences update}

Knowing that conclusions result from a zero-order TS model, a quadratic method can be used for determining the output of the rules $y\left(x_{n}\right)$. The optimization problem can be formulated as follows:

$$
y_{n+1}^{(j)}=y_{n}^{(j)}-\eta \cdot \frac{\partial E r}{\partial y_{n}^{(j)}}
$$

Where $\eta$ and $E_{r}$ respectively the learning rate and the quadratic criterion defined in (6). The proposed method for distance estimation should ensure a rule base interpretability so that properties of continuity, consistency and completeness are respected (Touati et al., 2006).

Once distances between nodes are estimated, a localization method based on Al-Kashi theory can be then implemented in order to determine the right coordinates in the environment. 


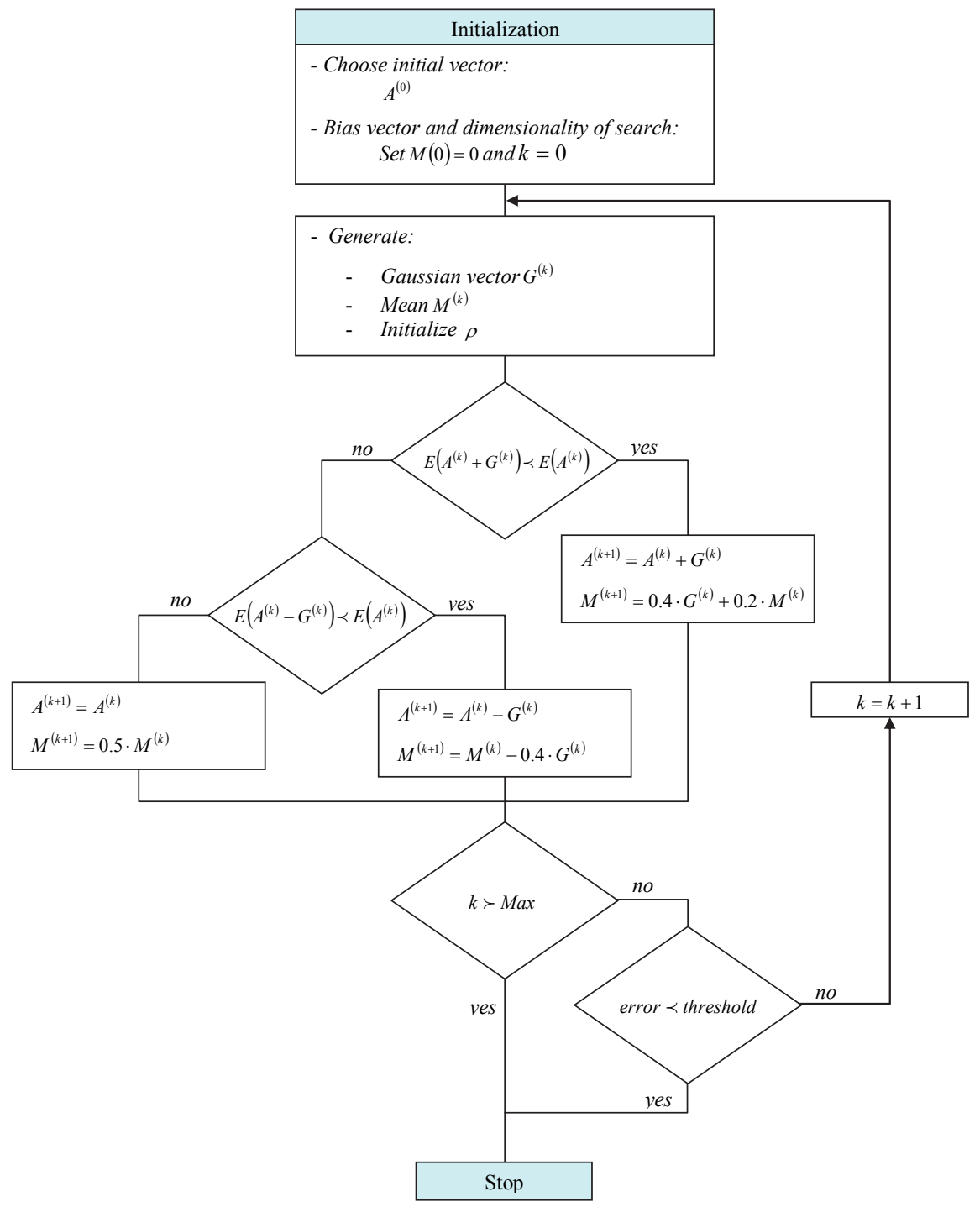

Fig. 4. Solis and Wets Pseudo-algorithm

In order to increase the optimization quality, rules conclusions are updated online using a stochastic gradient method.

\subsubsection{Al-Kashi theory for nodes localization}

Triangulation is the process of finding a distance to a point by calculating the length of one side of a triangle, given measurements of angles and sides of the triangle formed by that point and two other reference points. The implementation requires three reference points whose distances $d_{i}$, between nodes and the base are predefined during estimation phase. In 
this direction, Al-Kashi theory seems to be an efficient tool. The idea is to determine precisely the node position $M_{x}\left(x_{X}, y_{Y}\right)$, localized at distance $d_{1 x}$ from the base $B_{S 1}\left(x_{1}, y_{1}\right)$. In other words, this consists to determine the right orientation angles $\left(\alpha, \alpha_{0}, \beta\right)$, as it's illustrated on Fig.5.

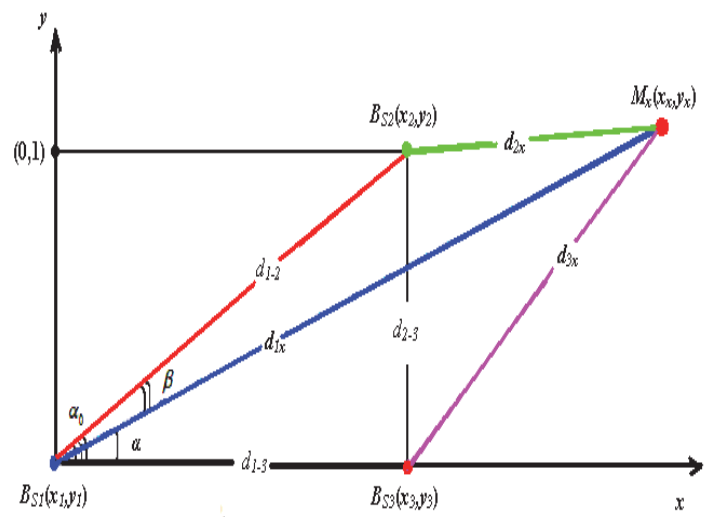

Fig. 5. Triangulation scheme

Thus, having information, i.e., positions and RSSI levels of each node $B_{S_{i}}\left(x_{i}, y_{i}\right)$, the distance between nodes $i$ and $j$ can be computed as follows:

$$
d_{i-j}=\sqrt{\left(x_{j}-x_{i}\right)^{2}+\left(y_{j}-y_{i}\right)^{2}}
$$

From Fig. 6, we can write:

$$
\alpha_{0}=a \cos \left(\frac{d_{1-3}}{d_{1-2}}\right)
$$

Based on Al-Kashi theory, we can write:

$$
d_{3 x}^{2}=d_{1 x}^{2}+d_{1-3}^{2}-2 d_{1 x} d_{1-3} \cos (\alpha)
$$

and,

$$
d_{2 x}^{2}=d_{1 x}^{2}+d_{1-2}^{2}-2 d_{1 x} d_{1-2} \cos (\beta)
$$

Thus:

$$
\alpha=a \cos \left(\frac{d_{1 x}^{2}+d_{1-3}^{2}-d_{3 x}^{2}}{2 d_{1 x} d_{1-3}}\right)
$$

and,

$$
\beta=a \cos \left(\frac{d_{1 x}^{2}+d_{1-2}^{2}-d_{2 x}^{2}}{2 d_{1 x} d_{1-2}}\right)
$$


The position of any supposed node can be computed according to some cases of angle $\beta$, so that:

$$
\beta=\left(\alpha-\alpha_{0}\right)
$$

Thus:

$$
\left\{\begin{array}{l}
x_{X}=( \pm) d_{1 x} \cos (\alpha) \\
y_{X}=( \pm) d_{1 x} \sin (\alpha)
\end{array}\right.
$$

To resume, knowing distances between nodes after implementing the fuzzy estimator, i.e., distance between mobile node $M_{x}\left(x_{X}, y_{Y}\right)$ and $B_{S 1}\left(x_{1}, y_{1}\right)$, we can determine the right position of $M_{x}\left(x_{X}, y_{Y}\right)$.

\section{Experimental setup}

LIASD-Wheelchair is an adjustable adults' powered wheelchair (Fig.6). It is suitable for indoor or outdoor use and implements wired and wireless networks for communication. The wireless communication is based on IEEE 802.11.4 standard (Touati et al., 2010).

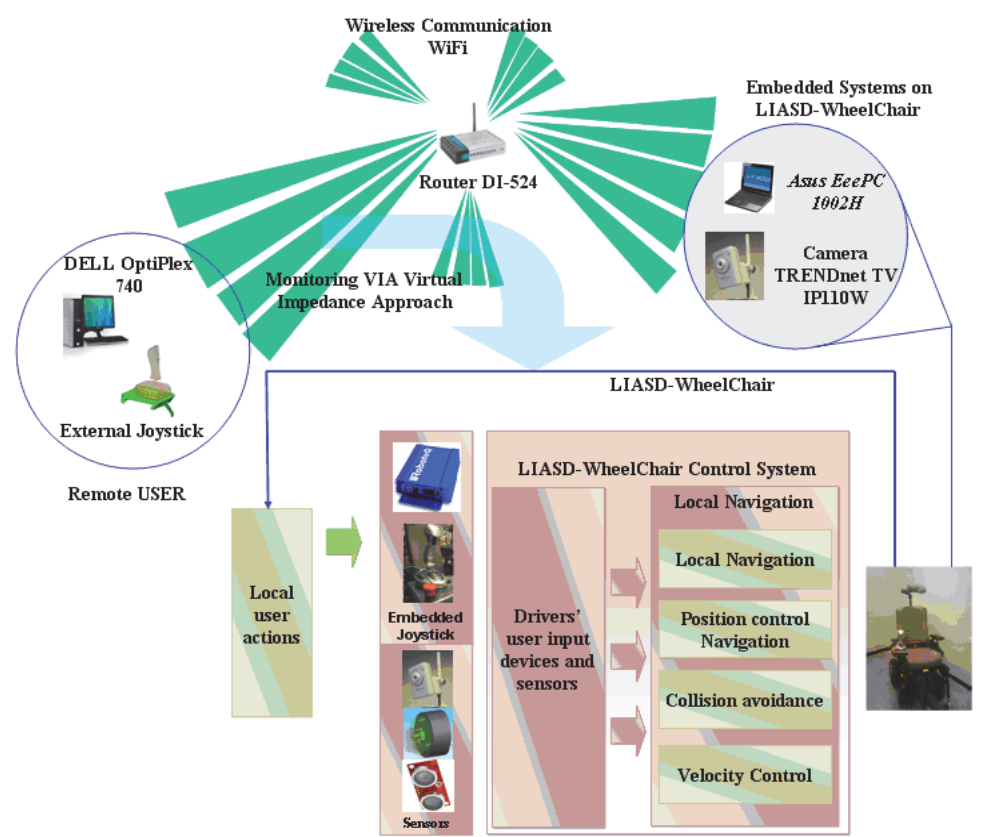

Fig. 6. Global structure of LIASD-WheelChair

The hardware architecture of LIASD-Wheelchair consists of sensory block, control architecture, and communication networks. The presented system includes two optical incremental encoders mounted to a motor, with resolution of 500 Counts per Revolution. Four ultrasonic rangefinders (US SFR08) are used to localize the wheelchair in the 
environment. They have a resolution of $3 \mathrm{~cm}$ and can identify obstacles between $3 \mathrm{~cm}$ and $6 \mathrm{~m}$. The US sensors interact with the computer via TCP/IP server board FMod-TCP DB using an I2C interface. In order to ensure navigation and anti-collision objectives a Wireless Internet Camera Server is mounted on the wheelchair headrest.

The communication network implements an I2C protocol for US sensors management via Fiveco card. USB and RS232 protocols are used to ensure communication between AX2550 controller and embedded laptop Asus-EeePC-1002H.

Knowing that the network architecture is based on an infrastructure mode, the remote user/wheelchair communication is ensured via an access point DI-524 which is able to transfer data at rates of $54 \mathrm{Mbps}$ and $2.4 \mathrm{GHz}$ through its wireless channels.

For the localization process, a WSN working on IEEE 802.15.4 compliant standard is deployed in the environment. Sensors are the latest generation of Motes from Crossbow Technology, called MICAz. They use the Chipcon CC2420 working on $2400 \mathrm{MHz}$, ZigBee ready radio frequency transceiver integrated with an Atmega128L micro-controller and serial flash memory. Fig.7 illustrates the MICAz sensor node with standard antenna and its bloc diagram.
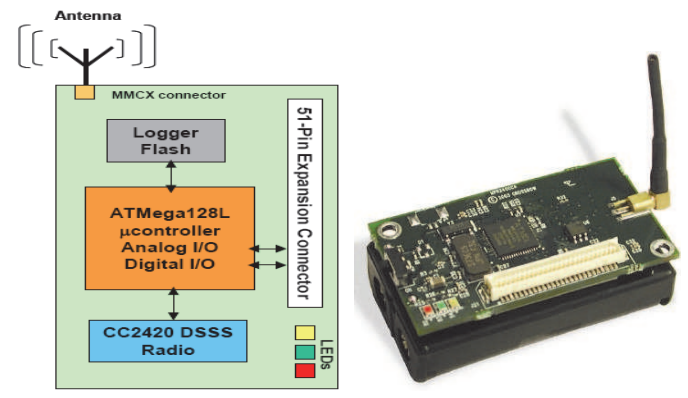

Fig. 7. MICAz sensor node and its architecture

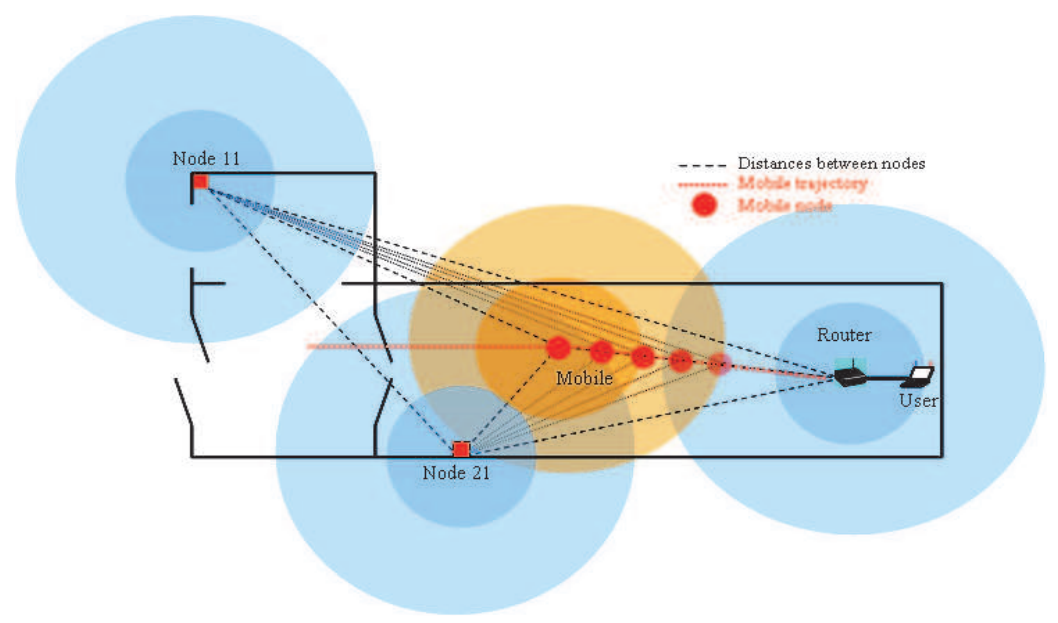

Fig. 8. LIASD-WheelChair moving in WSN environment. 


\section{Experimentation and results}

The proposed localization approach is implemented and tested on an intelligent wheelchair, called LIASD-WheelChair, developed at Computer Science and Artificial Intelligence Lab. LIASD-WheelChair operates in WSN environment. Four Crossbow MICAz nodes are implemented: Two anchors (nodes 11 and 21), one user node and one mobile node representing the wheelchair (Fig.8).

As it's illustrated on Fig.9, the FIS structure conception includes three MISO FIS subsystems, where, $I_{R S S I}=\left(I_{R S S I 11}, I_{R S S I 21}, I_{R S S I M}\right)$ and $d_{R S S I}=\left(d_{R S S I 11}, d_{R S S I 21}, d_{R S S I M}\right)$ represent respectively RSSI measures and distances between nodes and user node.

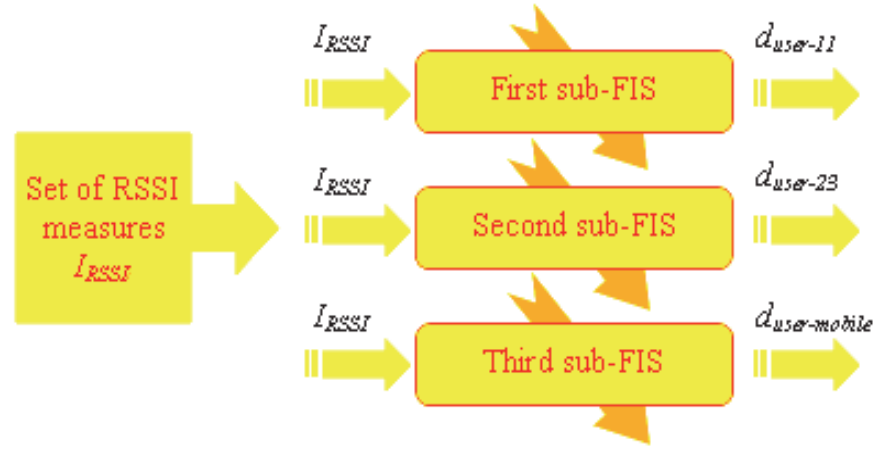

Fig. 9. FIS architecture

For each input variable space $I_{R S S I}$ partitioning, a set of triangular MFs is randomly initialized and the universe of discourse for each variable is divided into $k_{i}$ fuzzy sets.

Knowing that nodes 11 and 21 represent anchors nodes (Fig.8), the associated RSSI values should be approximately constant. Thus, distances between nodes $21-11$, user-21 and user-

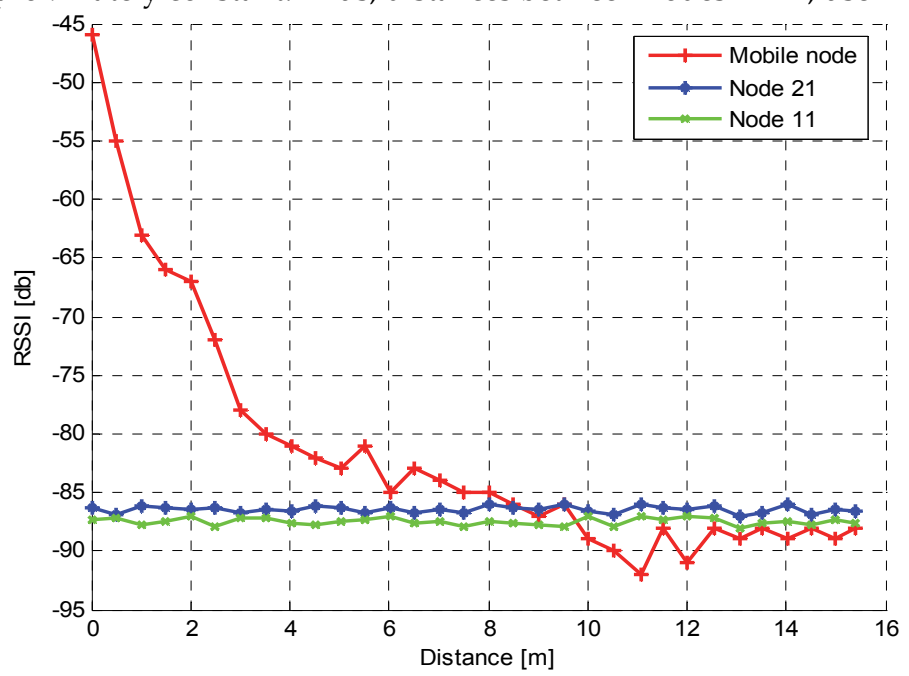

Fig. 10. RSSI measures vs. distances 
11 should approximately be a constant. This leads us to deal only with mobile node $M$, thus associated MISO FIS sub-systems (Third sub-FIS) (Fig.9).

The wheelchair moves in the environment according to specific desired trajectories. In the beginning, experimentation consists to collect a set of RSSI information from different nodes in order to conceive an initial FIS architecture. For this purpose, an initial database $\left(I_{R S S I i}, d_{R S S I i}\right)$ corresponding respectively to RSSI and distances, provided by a mobile node is built, and Fig.10 illustrates the obtained results concerning the whole nodes.

The learning error associated MFs optimization and conclusions update are illustrated respectively on Figures 11 and 12. The results show that the proposed algorithm for distance estimation is very efficient according to obtained error values. It ensures convergence directly at the first steps.

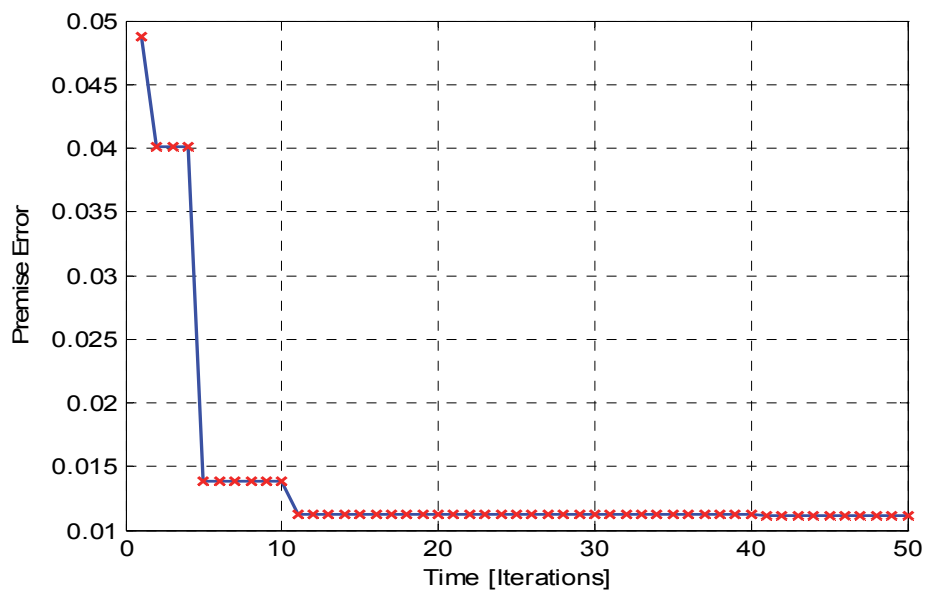

Fig. 11. Membership functions optimization

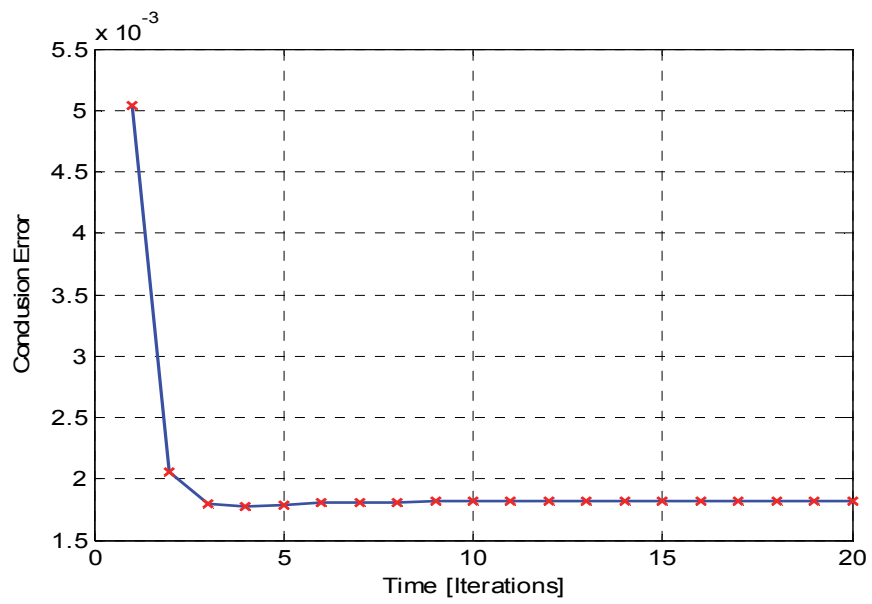

Fig. 12. Conclusions update 
In order to increase performances of the proposed fuzzy estimator, an online computation of distances is implemented according to Solis and Wets method. The obtained results which are illustrated on Fig.13, allows us to conclude that the approach can operates in real time.

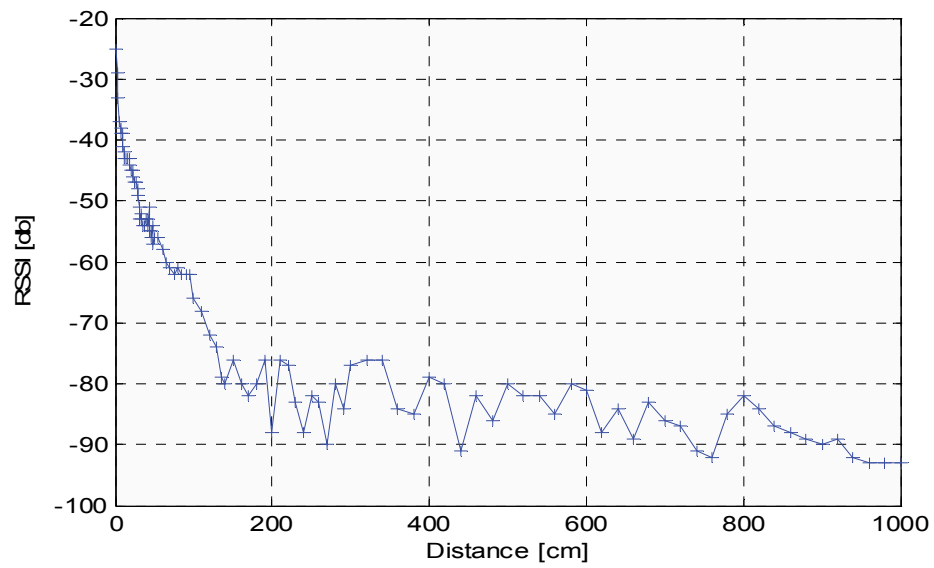

Fig. 13. RSSI measures vs. distance estimation

Fig.14 illustrates wheelchair trajectory operating in WSN environment according to $\mathrm{x}$ - and $\mathrm{y}$-axis.

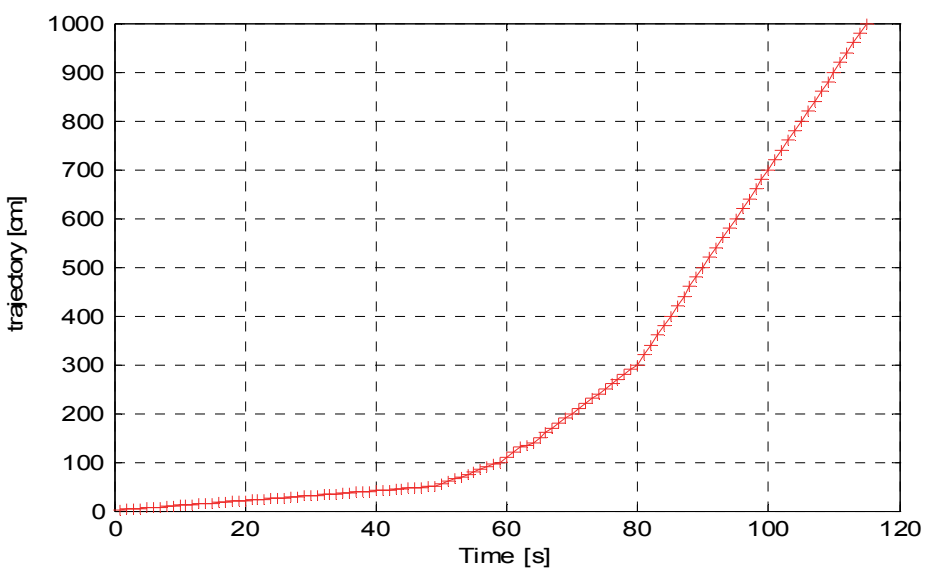

Fig. 14. FIS for online distance estimation

The analysis of obtained results shows that the proposed localization method can approximate nodes distances from RSSI measures and provided by sensor nodes (MICAz). In all cases, the use of fuzzy estimator generates a better estimation than LQI or generic models. Implementing an evolutionary strategy allows a better convergence speed according to classical algorithms requiring an additional phase for conflicts and redundancy treatment. This means that the proposed fuzzy estimator can be embedded on each node and implemented online for distances estimation between nodes. 


\section{Conclusion}

In this paper, we have proposed a WSN localization method based on IEEE 802.15.4 standard using fuzzy logic concepts with an evolutionary algorithm for parameters approximation and optimization. The approach allows approximating a realistic model for distance estimation from a set of RSSI measures and taking into account environment influences and signal failures as well as imprecise distances. The approach is based on a FIS optimization methodology which combines both a Rapid Prototyping mechanism and Solis and wets' mono-agent evolution strategy. The methodology allows, in term of probability, a randomized hill-climber with an adaptive step size and a global minima convergence of a coast function. It describes a class of local and global search algorithms with proofs of convergence in the limit of infinite search time. In contrast to the existing methods for FIS optimization methodology where a large number of parameters has to be tuned leading to significant computing times requiring high memory capacity, the proposed approach needs only a few rules for FIS knowledge base optimization, which leads to increased optimization convergence speed and avoids additional criteria such as rules simplification. The proposed approach takes into account an online and offline aspects and operates in two stages. In the first one, an evolutionary method based on Solis and Wets algorithm is implemented in conjunction with a PA mechanism to estimate online distances between nodes according to a set of RSSI measures. It's based on Glorennec's methodology for FIS data structure optimization and can be implemented off- or on-line and it proceeds as follows: A first set of rules is extracted automatically from training data using RP mechanism Thus, in conjunction with RP mechanism, an evolutionary algorithm based on Solis and Wets method for MFs optimization is then implemented. In the second stage, to increase the optimization quality, rules conclusions are updated online using a stochastic gradient method. In order to compute real positions, a triangulation method based on Al-Kashi theory is implemented using data derived from the second phase. In order to illustrate the efficiency of the proposed approach, experimentation on a smart wheelchair developed in our Lab. called LIASD-WheelChair has been performed.

\section{References}

Röhring, C. \& Spieker, S. (2009). Tracking of transport vehicles for warehouse management using a wireless sensor network. IEEE International Conference on Intelligent Robots and Systems, pp. 3260-3265, Nice, France

Vossiek, M.; Wiebking, L.; Gulden, P.; Wieghardt, J., Hoffmann, C. \& Heide, P. (2003). Wireless local positioning. Microwave magazine, vol.4, $\mathrm{n}^{\circ}$. 4, pp. 77-86

Tsung-Han, L. \& Hei-Ng, I. (2008). A microscopic examination of an rssi-signature-based indoor localization system. The 5th Workshop on Embedded Networked Sensors, Virginia, USA

Awad, A.; Frunzke, T. \& Dressler, F. (2007). Adaptive Distance Estimation and Localization in WSN using RSSI Measures. Proceeding of the 10th Euromicro-Conference on Digital System Design Architectures, Methods and Tools, pp. 471-478

Graefenstein, J. \& Bouzouraa, M.E. (2008). Robust Method for Outdoor Localization of a Mobile Robot Using Received Signal Strength in Low Power Wireless Networks. Proceeding of IEEE International Conference on Robotics and Automation, pp. 33-38, Pasadena, California 
Sugano, M. (2006). Indoor Localization System using RSSI Measurement of Wireless Sensor Network based on ZigBee Standard, Wireless and Optical Communications, ACTA Press, pp. 1-6

Cho, H.; Kang, M.; Park, J.; Park, B. \& Kim, H. (2007). Performance Analysis of Location Estimation Algorithm in ZigBee Networks Using Received Signal Strength. In 21 st International Conference on Advanced Information Networking and Applications Workshops, vol. 2, pp. 203-206, Niagara Falls, Ontario

Zadeh, L.A. (1965). Fuzzy sets. Information \& Control, 8 (3) 338-353, ISSN 0933-5846

Bezdek, J.C. (1981). Pattern recognition with fuzzy objective function algorithms. Plenum Press, ISBN:0306406713, New York

Dave, R.N. (1990). Fuzzy shell-clustering and applications to circle detection in digital images. Journal of General Systems, Vol.16, pp. 343-355, ISSN 0308-1079

Liang, C.Y. \& Su, J.P. (2003). A new approach to the design of a fuzzy sliding mode controller. Fuzzy Sets and Systems, 139(1), pp. 111-124, ISSN 0165-0114

Huang, L.; Ge, S.S. \& Lee, T.H. (2003). Fuzzy unidirectional force control of constrained robotic manipulators. Fuzzy Sets and Systems, 134(1), pp. 135-146, ISSN 0165-0114

Lei, S.; Pallazzolo, A.; Na, U. \& Kascak, A. (2001). Non-linear fuzzy logic control for forced large motions of spinning shafts. Journal of Sound and Vibration, Vol.235, N.3, pp. 435-449

Solis, F. \& Wets, J. (1981). Minimization by random search techniques. Mathematics of Operation Research, Vol.6, ISSN: 0364765X

Glorennec, P.Y. (1999). Learning algorithms for fuzzy inference systems. Hermes Ed. ISBN10: 2746200449

Hart, W.E. (1994). Adaptive Global Optimization with Local Search. dissertation at the University of California at San Diego La Jolla, CA, USA

Takagi, T. \& Sugeno, M. (1987). Fuzzy identification of systems and its applications to modeling and control. IEEE Transaction on Systems, Man and Cybernetics, Vol.1, pp. 116-132, ISBN 0018-9472

Touati, Y. \& Amirat, Y. (2006). Optimization and Design Methodology of Fuzzy Controller for Industrial Robotic Tasks. Proceeding of IEEE International Conference on Robotics and Automation, pp. 3666-3672, Orlando, Florida.

Touati, Y.; Aoudia, H. \& Ali-Chérif, A. (2010). Intelligent Wheelchair localization in wireless sensor network environment: A fuzzy logic approach. $5^{\text {th }}$ IEEE International Conference on Intelligent Systems, pp. 408-413, London, UK

Jiang, H.; Deng, H. \& He, Y. (2008). Determination of Fuzzy Logic Membership Functions Using Extended Ant Colony Optimization Algorithm. Fuzzy Systems and Knowledge Discovery Fourth International Conference on, 1, pp.581-585

Imai , H. \& Tanaka, A. (1998). A method of identifying influential data in fuzzy clustering. IEEE Transaction on Fuzzy Systems, vol. 6, n.1, pp.90-101

Ramon, N.; Castro, C.; Aguilar, L.T.; Castillo, O. \& Rodriguez, A. (2008). Optimizing Type-1 and Type-2 Fuzzy Logic Systems with Genetic Algorithms, Research in Computing Science, vol.39, pp.131-153

Castillo, O., Aguilar, L.T.; Cazarez, N. \& Cardenas, S. (2008). Systematic design of a stable type-2 fuzzy logic controller, Journal of Applied Soft Computing, vol. 8, no. 3, pp. 1274-1279 


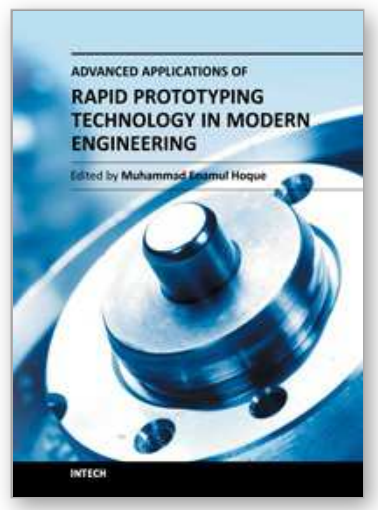

\section{Advanced Applications of Rapid Prototyping Technology in Modern Engineering}

Edited by Dr. M. Hoque

ISBN 978-953-307-698-0

Hard cover, 364 pages

Publisher InTech

Published online 22, September, 2011

Published in print edition September, 2011

Rapid prototyping (RP) technology has been widely known and appreciated due to its flexible and customized manufacturing capabilities. The widely studied RP techniques include stereolithography apparatus (SLA), selective laser sintering (SLS), three-dimensional printing (3DP), fused deposition modeling (FDM), 3D plotting, solid ground curing (SGC), multiphase jet solidification (MJS), laminated object manufacturing (LOM). Different techniques are associated with different materials and/or processing principles and thus are devoted to specific applications. RP technology has no longer been only for prototype building rather has been extended for real industrial manufacturing solutions. Today, the RP technology has contributed to almost all engineering areas that include mechanical, materials, industrial, aerospace, electrical and most recently biomedical engineering. This book aims to present the advanced development of RP technologies in various engineering areas as the solutions to the real world engineering problems.

\section{How to reference}

In order to correctly reference this scholarly work, feel free to copy and paste the following:

Touati Youcef, Aoudia Hania, Ali-Cherif Arab and Mohamed Demri (2011). Position Location Technique in Wireless Sensor Network Using Rapid Prototyping Algorithm, Advanced Applications of Rapid Prototyping Technology in Modern Engineering, Dr. M. Hoque (Ed.), ISBN: 978-953-307-698-0, InTech, Available from: http://www.intechopen.com/books/advanced-applications-of-rapid-prototyping-technology-in-modernengineering/position-location-technique-in-wireless-sensor-network-using-rapid-prototyping-algorithm

\section{INTECH}

open science | open minds

\section{InTech Europe}

University Campus STeP Ri

Slavka Krautzeka 83/A

51000 Rijeka, Croatia

Phone: +385 (51) 770447

Fax: +385 (51) 686166

www.intechopen.com

\section{InTech China}

Unit 405, Office Block, Hotel Equatorial Shanghai

No.65, Yan An Road (West), Shanghai, 200040, China

中国上海市延安西路65号上海国际贵都大饭店办公楼405单元

Phone: +86-21-62489820

Fax: $+86-21-62489821$ 
(C) 2011 The Author(s). Licensee IntechOpen. This chapter is distributed under the terms of the Creative Commons Attribution-NonCommercialShareAlike-3.0 License, which permits use, distribution and reproduction for non-commercial purposes, provided the original is properly cited and derivative works building on this content are distributed under the same license. 